\title{
EDitorial
}

One of the main university activities is research. This task is quite important for the College of Industrial Engineering, considering that the researching must be developed; it should be oriented to offer solutions to the productive sectors.

In this context emerges what is called the researching result transferring, which is one of the technological transferring expressions.

In order to develop the kind of research that is pointed; it must look for the approach between the university and the productive sector, in special the small companies.

In that sense, the faculties, doctoral students, graduate student, and undergraduate students are invited to realize the research that meets the characteristics previously appointed.

Ciencia, Tecnología e

Innovación para el desarrollo nacional 


\section{Modelo de perfil del actor político \\ Estudio del elemento humano en \\ organizaciones turbulentas}

\section{RESUMEN}

Existen organizaciones, denominadas partidos políticos, que influyen en el gobierno, empresas, sectores $y$, prácticamente, atraviesan todas las esferas de actividad de la sociedad. Sus miembros poseen evidentes fortalezas que les permiten navegar en ambientes turbulentos, con problemas indefinidos. Comparativamente, el profesional técnico muestra debilidades de interacción si el entorno es complejo y los problemas son blandos y no estructurados. A partir de la hipótesis de trabajo: "los activistas de los partidos políticos presentan un perfil semejante que les permite desenvolverse eficazmente en diversos entornos", se diseñó un constructo y modelo conceptual para identificar las variables que configuran su éxito.

El presente artículo muestra avances de la investigación empírica, intenta describir el perfil del activista político y sus fortalezas, las que se requieren para desenvolverse adecuadamente en ambientes cambiantes.

Palabras clave: Conocimiento, paradigma, matriz de datos, perfil político, Eros y Tanatos.

MODEL OF POLITICAL PLAYER'S PROFILE STUDY OF THE HUMAN ELEMENT IN TURBULENT ORGANIZATIONS

\section{ABSTRACT}

There are organizations, known as political parties, that influencing the government, businesses, industries, and virtually crossing all fields of society. Their members have strengths that allow them to play in turbulent environments with undefined problems. Otherwise, the professional technical has weaknesses to interact when the environment is complex and problems are soft unstructured. Since the working hypothesis, "the activists of political parties have a similar profile that allows them to act effectively in diverse environments", we design a construct and a conceptual model to identify the variables that shape the success of these.

This article shows the progress of empirical qualitative research, it attempts to describe the profile of political activist and their strengths, which are required to operate adequately in changing environments.

KEYWORDs: Knowledge, paradigm, data matrix political profile, Eros and Thanatos.

\section{MARCO CONCEPTUAL}

\section{Antecedentes filosóficos y académicos}

Dentro de los primeros antecedentes, se considera los estudios de los filósofos. Aristóteles afirma que el acceso de la verdad viene por el conocimiento del mundo sensible y define los tipos de conocimiento teórico y práctico; por otro lado, Platón considera que el mundo sensible es un reflejo de las ideas y diferencian opinión, que deviene de la imaginación, de las creencias y conocimiento, que derivan del entendimiento y la inteligencia. Hume considera que el conocimiento deviene de las impresiones o las experiencias sensibles, donde las ideas son simples asociaciones de estas experiencias.

En tiempos recientes, Morin afirma que la aptitud natural de la inteligencia para entender la complejidad del mundo, se desarrolla mediante la ubicación del conocimiento (informaciones) en un contexto y un conjunto.

Los antecedentes conceptuales se encuentran en la teoría X-Y de Douglas McGregor y en los conceptos de eficacia directiva de Peter Drucker. Recientemente, se considera la obra de Handy respecto a la naturaleza cambiante del trabajo y el sentido de la incertidumbre en el futuro, derivada de la paradoja de la certeza de pensamientos opuestos (continuidad y cambio), el trabajo sobre aprendizaje y solución de problemas por experiencias desarrollado por Kolb, Rubin y Maclntyre que plantean cuatro estilos de aprendizaje basado en las aptitudes de los directivos (divergente, asimilador, convergente y acomodador). El estudio de Senge sobre las organizaciones inteligentes define que el don o talento para el desarrollo de las cinco disciplinas de aprendizaje es una práctica que exige estudio y concentración. Peter Vaill amplía el aspecto humano del modelo sociotécnico, plantea que las organizaciones deben considerar, equilibradamente, el diseño técnico y el comportamiento humano. Su análisis relaciona la estructura de la tarea con las necesidades y las expectativas del individuo, concluye que la gestión debe considerar el sentido de la tarea y el significado del hombre para sí mismo, dentro de la pertinencia de los factores ambientales.

1 Magíster en Administración, Ingeniero Industrial, Profesor FII-UNMSM. Email: aacevedo@ speedy.com.pe.

2 Magíster en Dirección, Ingeniero Industrial, Profesora FISI-UNMSM. Email: klinares@speedy. com.pe

3 Magíster en Administración, Ingeniero Industrial, Decano FII-UNMSM. Email: orestescachay@ yahoo.es 